2014

\title{
National and Store Brand Advertising Strategies
}

Jenyi Chen

Cleveland State University, j.chen27@csuohio.edu

Stanko Dimitrov

Follow this and additional works at: https://engagedscholarship.csuohio.edu/bus_facpub

Part of the Advertising and Promotion Management Commons

How does access to this work benefit you? Let us know!

\section{Publisher's Statement}

This is a post-peer-review, pre-copyedit version of an article published in Journal of the Operational Research Society. The definitive publisher-authenticated version (Chen, J., Dimitrov, S. (2014). National and Store Brand Advertising Strategies. Journal of the Operational Research Society) is available online at: http://www.palgrave-journals.com/jors/journal/vaop/ncurrent/ full/jors201420a.html

\section{Original Published Citation}

Chen, J., Dimitrov, S. (2014). National and Store Brand Advertising Strategies. Journal of the Operational Research Society.

This Article is brought to you for free and open access by the Monte Ahuja College of Business at EngagedScholarship@CSU. It has been accepted for inclusion in Business Faculty Publications by an authorized administrator of EngagedScholarship@CSU. For more information, please contact library.es@csuohio.edu. 
Title: National and Store Brand Advertising Strategies

First Author: Jenyi Chen

Cleveland State University

Operations and Supply Chain Management Department

1860 E. 18th Street BU 545,

Cleveland, OH 44114

P: 12166874748

F: 12166879343

email: j.chen27@csuohio.edu

Second Author: Stanko Dimitrov

Department of Management Sciences

University of Waterloo

200 University Avenue West

Waterloo, Ontario Canada N2L 3G1

P: 15198884567 ext. 38966

F: 15197467252

email: sdimitro@uwaterloo.ca

\section{Statement of Contribution}

This work is motivated by the increase in the number of premium store brands over the past decade, now such store brands may be more expensive per unit volume than national brands. With this perspective, in this work we explore advertising and pricing decisions national and premium store brands can make. We develop an analytical game theoretic model using a quadratic advertisement cost function. Using this model we characterize the advertising and pricing decisions each of the brands will make under different market conditions. We find that in some cases only the national brand or the store brand will advertise, both brands will advertise, or no brand will advertise. In the case when pricing and advertising decisions are made in unison, we find that a national brand is better off free riding from the advertising efforts of the store brand. Alternatively when pricing and advertising decisions are made sequentially or separately, we find that either of the two brands may advertise as determined by market conditions. It is such insights that may not be exhibited in practice, that we hope will be considered by brand managers. 


\title{
National and Store Brand Advertising Strategies
}

\author{
November 25, 2013
}

\begin{abstract}
As the propensity of premium store brands increases, retailers must consider different ways to drive sales besides promotional strategies. With this in mind, we consider a national brand and a (premium) store brand co-existing in a market. Each brand has to decide the amount to invest in advertising its product and the prices to charge its customers, which can be determined separately or in unison. When either advertising expenditures or pricing decisions are set, each brand must keep in mind that the advertising efforts and revenue may spillover between the two brands, customers that intend to purchase the national brand may end up purchasing the store brand and vice versa. We derive an analytical model of the situations described and characterize equilibrium advertising decisions. We find that the characteristics of a premium store brand may depend on which marketing/promoting instrument (advertising or pricing) is the primary method for driving demand; and in some situations a national brand may be better off to not advertise at all and instead let the premium store brand carry out all of the advertising.
\end{abstract}

\section{Introduction}

In this paper we consider a national brand (NB), also referred to as a manufacturer, and a store brand (SB), also referred to as a retailer, within the same marketplace. We characterize the equilibrium advertising strategies of the NB and the $\mathrm{SB}$ as a function of market state. In particular, we consider whether the market has spillover demand or not, and whether there is profit sharing amongst the national and store brands. Though they have been around since the 1950s (Patti and Fisk, 1982), the majority of store brands still compete with national brands based on price using some form of promotion or pricing strategy. However, as the market share of private labels increases (Kumar and Steenkamp, 2007), more retailers are introducing premium store (house) brands (Huang and Huddleston, 2009) that may actually 
be more expensive than national brands (Karp, 2012). In these situations the SB, retailer, may actually want to advertise its product, and not let the NB, manufacturer, do all of the advertising. In this paper we first present the general model we consider. We then derive the advertising decisions each brand will make given that prices are fixed a priori, similarly the pricing decisions each brand will make given the advertising decisions are fixed a priory. Finally, we present the advertising and pricing decisions each brand will make if both decisions are made in unison.

We use a Stackelberg model, with the national brand as the first mover in this paper. As discussed in detail in Section 3, we consider both advertising and pricing decisions made by each of the brands. We assume the NB and SB consider only one product, or a single product category, and not a collection of products/categories as Erdem and Chang (2012) show there may be cross categorical affects for both store and national brand. In solving for the optimal advertising and pricing levels, we find situations where the store brand will want to advertise in conjunction with the national brand. Similarly, there are situations when the store brand will not want to advertise at all and let the national brand do all of the advertising. Surprisingly, we find cases when the national brand will want to let the store brand do all of the advertising. We also find that the characteristics of a premium store brand may depend on which primary method is used for marketing/promoition (advertising or pricing). These results provide managerial insight as to possible reactions by a national brand to a new premium store brand.

In the remainder of the paper we first discuss related work in Section 2. We then, in Section 3, introduce and formally discuss and solve the advertising/pricing models. In Section 4, we present some managerial insights gleaned from our analytical results and conclude the paper.

\section{Related Work}

While there is rich literature in studying the dynamics between national and store brands, for a comprehensive and excellent review, see Sethuraman (2009), that compiles and assesses results from analytical models with empirical evidences and credibility from practice executives, most of the attention is paid on the competitive basis since a SB product is often viewed as a clone (copycat or generic) to the NB's. Because of this underlying assumption on the inferiority of SB products, it generally limits the retailer's strategy space to whether to launch SB products or not (Horowitz, 2000; Raju et al., 1995), and if so, how the SB should promote (in price) to undercut the NB and thus gain market share given certain market structure and product characteristics (Mills, 1999; Raju et al., 1990; Sayman et al., 2002). 
On the other hand, from the incumbent manufacturer's (NB's) perspective, the strategic decisions are then often confined to how to deter the SB's entrance (Mills, 1999), or how to develop effective counter-strategy in order to differentiate the NB from the SB or defend leadership in quality (Choi and Coughlan, 2006; Narasimhan and Wilcox, 1998). With this paper we address not only pricing strategies a SB can employ, but also advertising strategies.

Most studies advocate the NB manufacturers to invest in advertising and developing new products, while discouraging the SB retailers from advertising but rather promoting low prices, as said by Webster (2000) "price is the dominant variable in many store brands' value proposition." Although earlier work glean interesting insights into market equilibriums in terms of the existence of the SB and its price-promoting strategy in response to the NB's actions, these recommendations are mainly based on the assumption of no sustainable strong SBs, usually established through brand advertising. This may have been true traditionally as Brester and Schroeder (1995) show that the estimates of the marginal effectiveness of advertising on generic meats are not significant. However, in recent years, premium SBs have been introduced by many retailers to achieve differentiation from other stores and are positioned on superior quality rather than price, e.g., "Archer Farms" at Target Corp and "Simple Truth" at Kroger Co (Karp, 2012). Levy and Gendel-Guterman (2012) provide a conceptual framework to show the importance of creating a strong SB through advertising and innovation. It was found empirically that advertising also has an indirect effect on the SB's perceived quality, a most important factor in influencing customer's purchase intention of the SB.

Despite the recently discovered importance of advertising SB products, we find very little analytical investigation into how the NB should advertise to influence purchase intention, facing the emergence of premium SBs as retailers gear up effort in brand advertising and store loyalty. Birwacker (2011) proposes that investing in holistic brand development programs benefits not only NB manufacturers but also progressive SB retailers to drive higher differentiation and lasting relationship with their shoppers. Karray and Zaccour (2005, 2006) propose an analytical model in this area that characterizes the equilibrium advertising decisions made by each brand when a NB partially covers a SB's advertising costs. Furthermore Birwadker (2011) suggests, to compete for consumers' dollars, joining forces or strategic partnerships between store and national brands may be formed in gaining shopper insights and identifying focused destination categories. Therefore, the effect of advertising can be either category building (also known as informative/complementary) or share stealing (persuasive/competitive) as documented by existing literature (Dubé and Manchanda, 2005; Roberts and Samuelson, 1988; Vilcassim et al., 1999).

Despite the rich literature in studying the strategic roles of advertising for firms facing 
competition, only a few papers examine the interaction between advertising and pricing decision. Vilcassim et al. (1999) propose an oligopoly model to econometrically analyze the dynamic pricing and advertising competition among firms and find empirically that firms compete on advertising but collaborate on pricing. Karray and Martín-Herrán (2008) study the relationship between the two decisions made sequentially through a game-theoretic model and find that in the case of competitive advertising, the pricing effects depends on the intensity of the competition and advertising. However, the target of their retailer's advertising is not on the SB product but rather the store itself so such complementary advertising always improves both the NB's and SB's demand; while in this paper, we consider premium store brands so their advertising campaigns may either be complementary or competitive. In a differential game setting, assuming symmetric price sensitivity for both the NB and SB for tractability, Karray and Martín-Herrán (2009) study the joint advertising and pricing decisions facing the two brands and find the retailer's best response to competitive advertising also depends on the intensity of pricing and advertising competition while the manufacturer always concedes on price and advertising under greater competition.

In this paper we employ a Stackelberg game-theoretic model to study the NB/SB (manufacturer/retailer) strategic advertising and pricing interaction, in the spirit of Karray and Martín-Herrán (2008). Making pricing and product selection decisions has been examined thoroughly in the above-mentioned earlier works, however in this work we examine both pricing and advertising decisions being made in unison or separately. Specifically, complementary and competitive advertising is incorporated into the model to evaluate the impact of the collaboration/competition level on the two parties' advertising and pricing decisions. Attention is given to what advertising and pricing strategy the market leader (the NB manufacturer) would adopt in anticipation of the response from the follower (the SB retailer) and the extent to which the two collaborate or compete. In addition to characterizing the optimal advertising and pricing decisions by the manufacturer and the retailer, we further consider and derive the conditions for the corner solutions in which only one channel member exerts efforts in advertising, and the conditions under which premium SB may possibly arise.

\section{Model and Analysis}

In this section we present the analytical model that determines the NB's and SB's best pricing and advertising strategies. In Section 3.1 we introduce the notation of the model and present the most general formulation. We start with analyzing special cases of the general formulation in Sections 3.2 and 3.3, in which we consider advertising decisions with pricing decisions given, and pricing decisions with advertising decisions given, respectively. Finally, 
in Section 3.4 we solve the general instance with both pricing and advertising decisions made in unison.

\subsection{Notation and Model Setup}

In the model we consider a national brand, $\mathrm{NB}$, and a store brand, $\mathrm{SB}$, each setting their own retail prices, $p_{i}$ with $i \in\{N, S\}$, where $p_{N}$ is the retail price of the NB and $p_{S}$ is the retail price of the $\mathrm{SB}^{1}$. Similarly both brands will set the amount of advertising effort to exert in order to increase market sizes, $A_{i}, i \in\{N, S\}$. As supported by the findings in Sudhir (2001) and Che et al. (2007), we model the manufacturer-retailer relationship using a manufacturer Stackelberg framework. In fact in addition to the two papers above, in a recent literature survey no paper was found to use Nash simultaneous moves (Sethuraman, 2009, page 8) in this setting.

The parameters of the model that are exogenously determined will now be discussed. As the NB is already established there is a pre-existing base market size $\underline{A_{N}}$ with neither the SB nor NB exerting any additional advertising effort. Without loss of generality, we assume the manufacturing costs of both products to be zero. A fraction $\beta \in(0,1)$ of all NB sales will go the SB as profit, and therefore the wholesale price of the NB product is $(1-\beta) p_{N}$. Though each brand is setting its own advertising decision, as the two brands are selling substitutable products, $\alpha_{i}$ measures the spillover effect of advertising effort from $i \in\{N, S\}$ to $j \in\{N, S\}: j \neq i,\left(\alpha_{S} \in(0,1)\right.$ and $\left.\alpha_{N} \in(-1,1)\right)$. For example, $\alpha_{N}$ is the advertising effect from NB to SB. The range of $\alpha_{N}$, is used to capture both complementary, $\alpha_{N}>0$, and competitive, $\alpha_{N}<0$, advertising scenarios. We do not consider $\alpha_{S}$ negative, as we do not model a national brand advertising directly against a store brand good.

Since pricing has an effect on the demand for goods of each brand, we introduce $a_{i}, i \in$ $\{N, S\}$ as the negative effect of price on the demand for the good sold by $i$. Similarly, we define $b_{i}$ as the positive effect the competitor's price, $j \in\{S, N\}$ has on demand for the good sold by $i \in\{N, S\}: i \neq j$. For example, $a_{N}$ measures the impact of $p_{N}$ on the demand for NB's product, similarly $b_{N}$ measure the impact of $p_{S}$ on the demand for NB's product. Finally, we use $c_{i}, i \in\{N, S\}$, to denote the marginal cost of advertising for each of the brands. As we will discuss below the advertising cost is assumed to be quadratic in the market size.

\footnotetext{
${ }^{1}$ Please see Appendix A for an exhaustive list of the notation used.
} 


\section{Profit Functions}

Using the notation introduced above, we formally present the profit functions we use in our model. We use $\pi_{i}$ to denote the profit of brand $i \in\{N, S\}$. We define $\pi_{N}$ as:

$$
\pi_{N}\left(A_{N}, p_{N}\right)=(1-\beta) p_{N}\left(A_{N}+\underline{A_{N}}+\alpha_{N} A_{S}-a_{N} p_{N}+b_{N} p_{S}\right)-c_{N}\left[\left(A_{N}+\underline{A_{N}}\right)^{2}-\underline{A_{N}}{ }^{2}\right] .
$$

For given values of the decision variables, $A_{N}$ and $p_{N},(1)$ is the profit function of the NB. $(1-\beta)$ is the proportion of all NB sales revenue that the NB retains. $p_{N}$ is the per unit price of the $\mathrm{NB}$, and $\left(\underline{A_{N}}+A_{N}+\alpha_{N} A_{S}-a_{N} p_{N}+b_{N} p_{S}\right)$ are the number of units sold as a function of the advertising efforts of both brands $\left(A_{N}+\alpha_{N} A_{S}\right)$, the base market size $A_{N}$, and the pricing strategies of both brands $b_{N} p_{S}-a_{N} p_{N}$. Note that we are assuming the cost of advertising is not linear but quadratically increasing in the difference of desired and base market sizes. While the quadratic functional form captures the diminishing marginal returns to advertising as is done in other works (Amrouche et al., 2008a,b; Dubé and Manchanda, 2005; Karray and Martín-Herrán, 2008, 2009; Vilcassim et al., 1999), we further generalize the cost function to take into account that the marginal return rate on advertising may be decreasing in the base market size $\underline{A_{N}}$, i.e., advertising may cost more for the manufacturer with high base market size $A_{N}$ to further expand the market. Given the actions of the NB, the profit function for the SB can be written as:

$$
\begin{aligned}
\pi_{S}\left(A_{S}, p_{S} \mid A_{N}, p_{N}\right)= & p_{S}\left(A_{S}+\alpha_{S}\left(A_{N}+\underline{A_{N}}\right)-a_{S} p_{S}+b_{S} p_{N}\right) \\
& +\beta p_{N}\left(A_{N}+\underline{A_{N}}+\alpha_{N} A_{S}-a_{N} p_{N}+b_{N} p_{S}\right) \\
& -c_{S}\left[\left(A_{S}+\alpha_{S}\left(A_{N}+\underline{A_{N}}\right)\right)^{2}-\left(\alpha_{S}\left(A_{N}+\underline{A_{N}}\right)\right)^{2}\right] .
\end{aligned}
$$

Given the advertising and pricing actions of the NB, the first term of the sum in (2) is the profit from all the SB sales. The second term is the fraction of the profit the SB receives from the NB. Finally, the last term is the cost of advertising decisions made by the SB. Because the advertising cost $c_{S}\left[\left(A_{S}+\alpha_{S}\left(A_{N}+\underline{A_{N}}\right)\right)^{2}-\left(\alpha_{S}\left(A_{N}+\underline{A_{N}}\right)\right)^{2}\right]$ is uniquely determined by the market size decision $A_{S}$ and vice versa, for the ease of explanation, henceforth we would use advertising level when referring to $A_{i}, i \in\{N, S\}$. Here we assume the SB may have an advantage as a follower to inherit a base market size of $\alpha_{S}\left(A_{N}+\underline{A_{N}}\right)$, which is proportional to the NB's post-advertising market size or irrelevant if $\alpha_{S}=0$.

In the remainder of this section we first consider two special cases of the general setup, first, in Section 3.2, pricing decisions are fixed and only advertising decisions are made and second, in Section 3.3, advertising decisions are fixed and only pricing decisions are made. We characterize equilibrium decisions in both cases and provide some managerial intuition for 
these results. We conclude this section by presenting the general case in Section 3.4. In the following derivations we omit some steps which we include for completeness in Appendix B.

\subsection{Advertising Only Case}

In this section we first consider the special case where the NB and SB prices are predetermined before making advertising decisions. As we model the advertising decisions of the national and store brands under a Stackelberg game-theoretic framework, using backward induction, it is straightforward to show the SB's best response function, $A_{S}^{*}\left(A_{N}\right)$, for a given $A_{N}$ value is as written in (3). Since (2) is concave in $A_{S}$, its first order condition (FOC) leads to the store brand's reaction function $A_{S}^{*}\left(A_{N}\right)$ :

$$
A_{S}^{*}\left(A_{N}\right)= \begin{cases}\frac{p_{S}+\beta \alpha_{N} p_{N}}{2 c_{S}}-\alpha_{S}\left(A_{N}+\underline{A_{N}}\right) & \text { if } \frac{p_{S}+\beta \alpha_{N} p_{N}}{2 \alpha_{S} c_{S}}>A_{N}+\underline{A_{N}} \\ 0 & \text { otherwise. }\end{cases}
$$

From (3), we note that $A_{S}^{*}$ is decreasing in $\alpha_{S}$ because the SB benefits from free-riding off of the advertising spillover from the NB, while $A_{S}^{*}$ is increasing in $\alpha_{N}$ due to the share of the increasing revenue from selling NB products. Substituting $A_{S}^{*}\left(A_{N}\right)$ into (1) gives a concave function, so the FOC determine the NB's optimal and equilibrium advertising decisions.

$$
A_{N}^{*}= \begin{cases}\frac{(1-\beta)\left(1-\alpha_{N} \alpha_{S}\right)}{2} \cdot \frac{p_{N}}{c_{N}}-\underline{A_{N}} & \text { if } p_{N}>\bar{p}_{N} \text { and } \frac{p_{S}}{p_{N}}>\phi \\ \frac{1-\beta}{2} \cdot \frac{p_{N}}{c_{N}}-\underline{A_{N}} & \text { if } p_{N}>\bar{p}_{N} \text { and } \frac{p_{S}}{p_{N}} \leq \phi \\ 0 & \text { if } p_{N} \leq \bar{p}_{N} \text { and } \frac{p_{S}}{p_{N}}>\phi \\ 0 & \text { otherwise }\end{cases}
$$

and

$$
A_{S}^{*}= \begin{cases}\frac{1}{2} \frac{p_{S}}{c_{S}}+\frac{\beta \alpha_{N}}{2} \frac{p_{N}}{c_{S}}-\alpha_{S} \frac{(1-\beta)\left(1-\alpha_{N} \alpha_{S}\right)}{2} \frac{p_{N}}{c_{N}} & \text { if } p_{N}>\bar{p}_{N} \text { and } \frac{p_{S}}{p_{N}}>\phi \\ 0 & \text { if } p_{N}>\bar{p}_{N} \text { and } \frac{p S}{p_{N}} \leq \phi \\ \frac{1}{2} \frac{p_{S}}{c_{S}}+\frac{\beta \alpha_{N}}{2} \frac{p_{N}}{c_{S}}-\alpha_{S} \frac{A_{N}}{c_{N}} & \text { if } p_{N} \leq \bar{p}_{N} \text { and } \frac{p}{p_{N}}>\phi \\ 0 & \text { otherwise }\end{cases}
$$

where $\bar{p}_{N}=\frac{2 c_{N}}{(1-\beta)\left(1-\alpha_{N} \alpha_{S}\right)} \underline{A_{N}}$ and $\phi=(1-\beta) \alpha_{S}\left(1-\alpha_{N} \alpha_{S}\right) \frac{c_{S}}{c_{N}}-\beta \alpha_{N}$. The above four cases correspond to one interior optimal solution $\left(A_{N}^{*}>0\right.$ and $\left.A_{S}^{*}>0\right)$, two corner solutions $\left(A_{N}^{*}>0\right.$ and $\left.A_{S}^{*}=0\right) ;\left(A_{N}^{*}=0\right.$ and $\left.A_{S}^{*}>0\right)$, and one degenerate solution $\left(A_{N}^{*}=A_{S}^{*}=0\right)$. From the conditions for each case, it can be concluded that: first, for the NB to launch any advertising campaign, the price of the NB product must exceed some threshold, $\vec{p}_{N}$, which is increasing (in weak sense, i.e., non-decreasing) in all parameters $c_{N}, A_{N}, \beta, \alpha_{N}$, and $\alpha_{S}$ when the SB's advertising is complementary, i.e., the spillover effect with $\alpha_{N}>0$. Therefore, the 
NB will not advertise if any combination of the following scenarios holds: high advertising cost, large base market, high fraction of profit going to the retailer, or high spillover rates. For the spillover rates, while the free-riding effect of $\alpha_{N}$ is intuitive, it does not seem obvious that high NB-to-SB spillover rate $\alpha_{S}$ also discourages the NB from advertisement. This is the case because high $\alpha_{S}$ reduces the SB's advertising effort, which means less spillover from the SB advertisement toward the NB product. On the other hand, when the SB's advertising is competitive, $\alpha_{N}<0$, the price threshold $\vec{p}_{N}$ is lower, implying the NB would start advertising and invest more in advertisement as competition becomes more intense.

Second and independently, for the retailer to invest in advertising the SB product, the price ratio $p_{S} / p_{N}$ must be high enough, greater than the threshold $\phi$, which is increasing in $c_{S} / c_{N}$ and $\alpha_{S}$ (assuming $\alpha_{N} \alpha_{S} \leq 0.5$ ) while decreasing in $\beta$ and $\alpha_{N}$ with complementary SB advertising. If advertising is one of the key indicators of a premium $\mathrm{SB}$, then a premium $\mathrm{SB}$ should possess any combination of the following characteristics: low costs of advertising ratio $c_{S} / c_{N}$ (ease of advertising in comparison with the NB), low NB advertising spillover (unique NB features), high fraction of the NB sales revenue to the retailer (mature NB product so the retailer has more bargaining power over the NB), and high SB advertising spillover (high substitutability of SB to NB). When the $\mathrm{SB}$ advertising becomes competitive, $A_{N}^{*}$ increases to stay competitive, while $A_{S}^{*}$ decreases to free-ride from the gain of a higher $A_{N}^{*}$ as otherwise a SB would reduce its revenue from selling fewer NB goods.

The non-trivial solutions of $A_{S}^{*}$, (5), provide a different aspect in explaining the SB's advertising strategy: the first term $p_{S} / c_{S}$ corresponds to the effort for its own SB product while the second term $\beta \alpha_{N} p_{N} / c_{S}$ corresponds to the effect of NB sales due to the share $\beta$ of all NB revenue and advertising spillover $\alpha_{N}$. When both SB and NB advertise, the advertising effort is adjusted down by spilled efforts from the NB's advertisement $\alpha_{S}$ (1$\beta)\left(1-\alpha_{N} \alpha_{S}\right) p_{N} / c_{N}$, and when only the SB advertises the benefit is adjusted by the base market size.

\subsection{Pricing Only Case}

In this section we consider the case in which for given advertising expenditures of the NB and $\mathrm{SB}$, each may only maximize their expected profit by setting their pricing strategies, $p_{N}$ and $p_{S}$ respectively. Carrying out backward induction we can show that (2) is concave in $p_{S}$ for a given $p_{N}$ and fixed advertising strategies. Determining the optimal SB price for a given $p_{N}$ leads to:

$$
p_{S}^{*}\left(p_{N}\right)=\frac{A_{S}+\alpha_{S}\left(A_{N}+\underline{A_{N}}\right)+\left(b_{S}+\beta b_{N}\right) p_{N}}{2 a_{S}} .
$$


It can be readily seen from (6) that first, the SB's best response pricing decision $p_{S}^{*}\left(p_{N}\right)$ is increasing in the market size (or equivalently, marketing efforts made by itself and spilled from the NB). Second, $p_{S}^{*}\left(p_{N}\right)$ is linearly increasing in $p_{N}$ because of competing effects $b_{S}$ and $\beta b_{N}$. We can then substitute $p_{S}^{*}\left(p_{N}\right)$ in (1) and find the equilibrium NB price, $p_{N}^{*}$, via FOCs:

$$
p_{N}^{*}=\frac{A_{N}+\underline{A_{N}}+\alpha_{N} A_{S}+\frac{b_{N}}{2 a_{S}}\left[A_{S}+\alpha_{S}\left(A_{N}+\underline{A_{N}}\right)\right]}{2\left[a_{N}-\frac{b_{N}}{2 a_{S}}\left(b_{S}+\beta b_{N}\right)\right]} .
$$

The form of $p_{N}^{*}$ in (7) shows that it is increasing in both advertising efforts: the first part of the numerator $\left(A_{N}+\underline{A_{N}}+\alpha_{N} A_{S}\right)$ is the direct effect from self, NB, advertising and $\mathrm{SB}$ spillover efforts, while the second part $\frac{b_{N}}{2 a_{S}}\left[A_{S}+\alpha_{S}\left(A_{N}+\underline{A_{N}}\right)\right]$ reflects the indirect effect from the SB pricing strategy influenced by the SB advertising and NB advertising spillover. In the denominator, the sensitivity of NB demand to NB price is dampened from $a_{N}$ to $a_{N}-\frac{b_{N}}{2 a_{S}}\left(b_{S}+\beta b_{N}\right)$ due to the competing price of the $\mathrm{SB}, p_{S}$, in (5) that is also increasing $p_{N}$. We may find $p_{S}^{*}$ by substituting (7) into (6), which we omit in this exposition. Note that to ensure the concavity of the NB's profit function, (7) is only valid in the case that:

$$
a_{N}>\frac{b_{N}}{2 a_{S}}\left(b_{S}+\beta b_{N}\right) .
$$

Inequality (8) requires the direct demand sensitivity $a_{N}$ to be not totally offset by the indirect competing effect $\frac{b_{N}}{2 a_{S}}\left(b_{S}+\beta b_{N}\right)$. Should the downward slope $a_{N}$ be overly compensated and become upward sloping (inequality (8) is violated meaning the profit function becomes convexly increasing), it is then to the NB's advantage to set $p_{N}^{*}$ as high as possible to gain infinite profit, which is not a well-defined scenario. Due to this seemingly degenerate scenario, we do not consider it in detail in this paper.

As pricing is a focal point of this subsection, we glean further insights by investigating the relative magnitude of the two prices, $p_{N}^{*}$ and $p_{S}^{*}$. To have $p_{N}^{*}>p_{S}^{*}$ as most commonly seen, the following relationship must hold:

$$
\frac{A_{N}+\underline{A_{N}}+\alpha_{N} A_{S}}{A_{S}+\alpha_{S}\left(A_{N}+\underline{A_{N}}\right)}+\frac{b_{N}}{2 a_{S}}>\frac{2\left[a_{N}-\frac{b_{N}}{2 a_{S}}\left(b_{S}+\beta b_{N}\right)\right]}{2 a_{S}-b_{S}-\beta b_{N}} .
$$

The left hand side of (9) is the advertising effort ratio measuring the NB's effort with respect to the SB's with the adjustment $\left(b_{N} / 2 a_{S}\right)$ due to price competition. The right hand side measures the NB's demand sensitivity in price with respect to the SB's. The conditions in which this inequality is violated so that $p_{N}^{*} \leq p_{S}^{*}$ characterize a premium SB. From (9) we 
determine that a premium store brand will exists, for a set of fixed advertising decisions in the complementary advertising setting, if: the spillover rate is high from NB to SB $\left(\alpha_{S}\right)$ and a powerful retailer $(\beta)$, maybe due to a mature NB product, and the spillover rate is low from SB to $\mathrm{NB}\left(\alpha_{N}\right)$, perhaps due to some unique properties of the SB product.

As we allow for competitive advertising, i.e., $\alpha_{N}<0$, we observe that in this case there is a theoretical possibility for $p_{N}^{*}$ to be negative or zero. However, for this to occur, $A_{N}+\underline{A_{N}}<\left|\alpha_{N}\right| A_{S}$, which means the SB must put forth so much advertising effort that it advertises more than the NB and the base market size and effectively advertises the NB out of existence. Though theoretically feasible, we know of no such SB that has so much market power that it can ever impact the NB price to such a great extent.

\subsection{General Case}

This section considers the general case in which each brand determines its optimal advertising and pricing decisions in tandem. We assume that the NB sets its pricing and advertising decisions before the SB. Through backward induction, we find the best response functions of the SB, i.e., $A_{S}^{*}\left(A_{N}, p_{N}\right)$ and $p_{S}^{*}\left(A_{N}, p_{N}\right)$. We will then substitute the best response functions of the SB into the NB's profit function to determine the NB's optimal adverting and pricing levels, i.e., $A_{N}^{*}$ and $p_{N}^{*}$.

We first start with the SB's best response functions by considering its FOCs with respect to $A_{S}$ and $p_{S}$ to find $A_{S}^{*}\left(A_{N}, p_{N}\right)$ and $p_{S}^{*}\left(A_{N}, p_{N}\right)$ :

$$
\begin{gathered}
A_{S}^{*}\left(A_{N}, p_{N}\right)= \begin{cases}\frac{b_{S}+\beta b_{N}+2 \beta a_{S} \alpha_{N}}{4 a_{S} c_{S}-1} p_{N}-\alpha_{S}\left(A_{N}+\frac{\left.A_{N}\right)}{0}\right. & \text { if } \frac{b_{S}+\beta b_{N}+2 \beta a_{S} \alpha_{N}}{\alpha_{S}\left(4 a_{S} c_{S}-1\right)} p_{N}>A_{N}+\underline{A_{N}} \\
\text { otherwise. }\end{cases} \\
p_{S}^{*}\left(A_{N}, p_{N}\right)= \begin{cases}\frac{\beta \alpha_{N}+2 b_{S} c_{S}+2 \beta b_{N} c_{S}}{4 a_{S} c_{S}-1} p_{N} & \text { if } \frac{b_{S}+\beta b_{N}+2 \beta a_{S} \alpha_{N}}{\alpha_{S}\left(4 a_{S} c_{S}-1\right)} p_{N}>A_{N}+\underline{A_{N}} \\
0 & \text { otherwise. }\end{cases}
\end{gathered}
$$

To ensure optimality of (10) and (11) the second order optimality conditions (SOCs) must be satisfied, i.e., the Hessian of (2) must be negative semidefinite, equivalently $4 a_{S} c_{S}>1$ must hold.

Substituting the SB's best response functions (10) and (11) into (1), and we find the Hessian of the NB's profit function as

$$
\begin{aligned}
& \nabla^{2} \pi_{N}\left(A_{N}, p_{N} \mid A_{S}^{*}\left(A_{N}, p_{N}\right), p_{S}^{*}\left(A_{N}, p_{N}\right)\right) \\
& =\left[\begin{array}{cc}
-2 c_{N} & \left(1-\alpha_{N} \alpha_{S}\right)(1-\beta) \\
\left(1-\alpha_{N} \alpha_{S}\right)(1-\beta) & -2(1-\beta)\left(a_{N}-\frac{\alpha_{N}\left(b_{S}+b_{N} \beta+2 a_{S} \alpha_{N} \beta\right)+b_{N}\left(\alpha_{N} \beta+2 b_{S} c_{S}+2 b_{N} \beta c_{S}\right)}{4 a_{S} c_{S}-1}\right)
\end{array}\right] .
\end{aligned}
$$


To ensure that (12) is negative semidefinite, and that the $p_{N}^{*}$ and $A_{N}^{*}$ we find using FOCs are maximums, the following condition must hold as a lower bound on $c_{N}$ :

$$
c_{N} \geq \frac{\left(1-\alpha_{N} \alpha_{S}\right)^{2}(1-\beta)}{4\left(a_{N}-\frac{\alpha_{N}\left(b_{S}+b_{N} \beta+2 a_{S} \alpha_{N} \beta\right)+b_{N}\left(\alpha_{N} \beta+2 b_{S} c_{S}+2 b_{N} \beta c_{S}\right)}{4 a_{S} c_{S}-1}\right)} .
$$

To have a valid bound, the denominator of the RHS (right hand side) must be positive, i.e., $a_{N}>\alpha_{N} \frac{\left(b_{S}+b_{N} \beta+2 a_{S} \alpha_{N} \beta\right)}{4 a_{S} c_{S}-1}+b_{N} \frac{\alpha_{N} \beta+2 b_{S} c_{S}+2 b_{N} \beta c_{S}}{4 a_{S} c_{S}-1}$. This requires the direct demand sensitivity to be not totally offset by the indirect competing advertising effect $\alpha_{N} \frac{\left(b_{S}+b_{N} \beta+2 a_{S} \alpha_{N} \beta\right)}{4 a_{S} c_{S}-1}$ and competing pricing effect $b_{N} \frac{\alpha_{N} \beta+2 b_{S} c_{S}+2 b_{N} \beta c_{S}}{4 a_{S} c_{S}-1}$. Analogous to inequality (8) in the pricing-only case, except for the additional competing advertising effect, should the downward slope $a_{N}$ be overly compensated and become upward sloping, NB then sets $p_{N}^{*}$ as high as possible to gain infinite profit, which is not a well-defined scenario. We now find $p_{N}^{*}$ and $A_{N}^{*}$ of the NB using the FOCs $\frac{\partial \pi_{N}}{\partial A_{N}}=0$ and $\frac{\partial \pi_{N}}{\partial p_{N}}=0^{2}$ :

$$
\begin{aligned}
& \frac{\partial \pi_{N}}{\partial A_{N}}=0 \\
& \Longrightarrow p_{N}^{*}\left(A_{N}\right)= \frac{2 c_{N}}{(1-\beta)\left(1-\alpha_{N} \alpha_{S}\right)}\left(A_{N}+\underline{A_{N}}\right) \quad \beta, \alpha_{S}, \alpha_{N} \in(0,1), \\
& \frac{\partial \pi_{N}}{\partial p_{N}}=0 \\
& \triangle \Delta\left(A_{N}+\underline{A_{N}}\right)=0 \quad \text { after substituting the value of } p_{N}^{*}\left(A_{N}\right) .
\end{aligned}
$$

The functional form of $p_{N}^{*}\left(A_{N}\right),(14)$, shows a linear relationship between $p_{N}^{*}$ and $A_{N}$. The optimal form of $A_{N}^{*}$, shown in (15), has $A_{N}^{*}=-\underline{A_{N}}$ as $\Delta$ is a constant with respect to $p_{N}$ and $A_{N}$. This means that no interior solution exists to $\pi_{N}\left(A_{N}, p_{N} \mid A_{S}^{*}\left(A_{N}, p_{N}\right), p_{S}^{*}\left(A_{N}, p_{N}\right)\right)$ as we require the advertising effort exerted, $A_{N}$, and the base market size, $A_{N}$, to be non-negative. Therefore, as $A_{N} \in[0, \infty), A_{N}^{*}=0$ or $A_{N}^{*}$ is unbounded. However, as $\pi_{N}\left(A_{N}, p_{N} \mid A_{S}^{*}\left(A_{N}, p_{N}\right), p_{S}^{*}\left(A_{N}, p_{N}\right)\right)$ is concave, guaranteed by (13) and we identified at least one interior stationary point, then for all values of $A_{N}>-\underline{A_{N}}, \pi_{N}\left(A_{N}, p_{N} \mid A_{S}^{*}\left(A_{N}, p_{N}\right), p_{S}^{*}\left(A_{N}, p_{N}\right)\right)$ is decreasing. This means that the optimal feasible advertising effort for the NB is $A_{N}^{*}=0$. The fact that $A_{N}^{*}=0$ may be comforting as the only other option is to have $A_{N}^{*} \rightarrow \infty$, which would indicate the NB could turn its first mover advantage into a "money pump."

The result in this section may seem counter intuitive as NBs still advertise in the presence of SBs. However, as the special cases in Sections 3.2 and 3.3 indicate, if either the the

\footnotetext{
${ }^{2}$ Please see Appendix B for details
} 
pricing or advertising decisions are fixed, then the other decision may not necessarily be trivial (zero). This may indeed be the case as in some organizations pricing and advertising decisions may be made sequentially as is indicated by Eastlack and Rao (1986) in the case of V-8. Alternatively, there may be externalities that force firms, national brands included, to advertise as discussed by McDonald and Wren (2012), we do not model such externalities in this paper.

We conclude this section by discussion the implications of our results. From (10) and (11) we note that $A_{S}^{*} \neq 0$ and $p_{S}^{*}<p_{N}^{*}$ if and only if $\frac{\beta \alpha_{N}+2 b_{S} c_{S}+2 \beta b_{N} c_{S}}{4 a_{S} c_{S}-1}<1$. However, as noted previously a premium store brand may actually set $p_{S}^{*}$ such that $p_{S}^{*} \geq p_{N}^{*}$, which implies $\frac{\beta \alpha_{N}+2 b_{S} c_{S}+2 \beta b_{N} c_{S}}{4 a_{S} c_{S}-1} \geq 1$. This occurs if $\beta$ is large, i.e., SB receives a larger fraction of NB sales, in the case of strong SB market power. Similarly when $\alpha_{N}$ is large, i.e., there is large spillover from NB advertising to SB demand, this may occur in the case of strong substitutes between SB and NB goods. To summarize, though the NB does not advertise and the SB may set $p_{S}^{*} \geq p_{N}^{*}$ in the general case of our model, we discussed that corporations may still advertise and price if these decisions are made in sequence or separately from one another.

\section{Insight and Conclusion}

This work adds to the literature on advertising and pricing interplay that exists between a national brand and a store brand good. With the strong rise in store brand market share and the propensity of stores to launch premium store brands, we believe the managerial insights for both a retailer, store brand, and a manufacturer, national brand, will be of great interest. Table 1 summarizes the main contributions of this article using a stylized model. When only advertising decisions are considered, we found that premium store brands strive for a low NB spillover rate and a high SB spillover rate (or low competitive effect from SB advertising); while when only considering pricing decisions, however, we found that the opposite conditions are preferred, i.e., high NB and low SB spillover (high with SB competition) rates encourage premium store brands. It is important to note that whether the SB advertising is competitive or complementary has a deciding effect on how the retailer's bargaining power

over the wholesale price would influence the advertising and pricing strategies. Hence the characterization of a premium store brand depends on which marketing/promotion instrument (advertising or pricing) is the primary method for driving the demand. In the case when both methods are simultaneously utilized, the national brand manufacturer always exerts no advertising effort and free-rides the retailer. However, it may not be the case that both pricing and advertising decisions are made simultaneously, but rather a repeated/sequential process is used to determine the final equilibrium decisions. 


\begin{tabular}{|c|c|c|}
\hline Section & Decisions & Results \\
\hline$\S 3.2$ & Advertising & $\begin{array}{l}\text { NB advertises iff } p_{N}>\bar{p}_{N} \text { while SB advertises iff } \frac{p_{S}}{p_{N}}>\phi \text {. } \\
\text { Premium SB will exert advertising effort } A_{S}^{*} \text { if it has: } \\
\text { - low advertising cost ratio } \frac{c_{S}}{c_{N}} \\
\text { - low NB spillover } \alpha_{S} \\
\text { - for } \alpha_{N}>0 \text {, high SB complementary advertising } \alpha_{N} \\
\text { - for } \alpha_{N}<0 \text {, low SB competing advertising } \alpha_{N} \\
\text { - high retail margin } \beta \text { when } \frac{c_{S}}{c_{N}}>\frac{-\alpha_{N}}{\alpha_{S}\left(1-\alpha_{N} \alpha_{S}\right)} \text {; or low } \\
\quad \beta \text { otherwise }\end{array}$ \\
\hline$\S 3.3$ & Pricing & $\begin{array}{l}p_{S}^{*}>p_{N}^{*} \text { iff } \frac{A_{N}+A_{N}+\alpha_{N} A_{S}}{A_{S}+\alpha_{S}\left(A_{N}+\underline{A_{N}}\right)}+\frac{b_{N}}{2 a_{S}}<\frac{2\left[a_{N}-\frac{b_{N}}{2 a_{S}}\left(b_{S}+\beta b_{N}\right)\right]}{2 a_{S}-b_{S}-\beta b_{N}} \text { so } \\
\text { premium SB has: } \\
\text { - high NB spillover } \alpha_{S} \\
\text { - for } \alpha_{N}>0 \text {, low SB complementary advertising } \alpha_{N} \\
\text { - for } \alpha_{N}<0 \text {, high SB competing advertising } \alpha_{N} \\
\text { - high retail margin } \beta\end{array}$ \\
\hline$\S 3.4$ & $\begin{array}{l}\text { Advertising } \\
\text { \& Pricing }\end{array}$ & $\begin{array}{l}\text { NB never advertises so } A_{N}^{*}=0<A_{S}^{*} \text { and } p_{N}^{*}>p_{S}^{*} \text { iff } \\
\frac{\beta \alpha_{N}+2 b_{S} c_{S}+2 \beta b_{N} c_{S}}{4 a_{s} c_{S}-1}<1 \text {. Premium SB will price } p_{S}^{*}>p_{N}^{*} \text { if } \\
\text { it has: } \\
\text { - for } \alpha_{N}>0 \text {, high SB complementary advertising } \alpha_{N} \\
\text { - for } \alpha_{N}<0 \text {, low SB competing advertising } \alpha_{N} \\
\text { - high retail margin } \beta \text { when }-\alpha_{N}<2 b_{N} c_{S} \text {; or low } \beta \\
\text { otherwise }\end{array}$ \\
\hline
\end{tabular}

Table 1: A summary of the main results.

In the future, using retail data, it will be interesting to develop empirical methods to identify favorable market conditions for a premium store brand. In addition to this empirical question, some analytical questions remain unanswered. In particular, this article assumes that the advertising cost function is quadratic. This is a fine analytical assumption with diminishing return on investment, but in practice there might exist some other function governing the cost-demand relationship, such as an "S-shaped" function. Using historical 
retail data, we would like to derive particular functional forms for the cost-demand function and derive the optimal decisions and compare them to what occurs in practice. Finally, we would also like to extend our model to take into account the case in which a national and a store brand make decisions simultaneously, i.e., there is no leader-follower dominance.

As one can see, there are quite a few open areas of research in this field. However, we think that this work is a necessary step to help retailers make more informed store brand decisions and national brand manufacturers be better prepared to respond to the introduction of additional store brands.

\section{References}

Amrouche, N., Martín-Herrán, G., and Zaccour, G. (2008a). Feedback Stackelberg equilibrium strategies when the private label competes with the national brand. Annals of Operations Research, 164(1):79-95.

Amrouche, N., Martín-Herrán, G., and Zaccour, G. (2008b). Pricing and Advertising of Private and National Brands in a Dynamic Marketing Channel. Journal of Optimization Theory and Applications, 137(3):465-483.

Birwadker, S. (2011). Store brands vs. national brands: Strategies for success. Technical report, Landor Associates, landor.com.

Brester, G. W. and Schroeder, T. C. (1995). The Impacts of Brand and Generic Advertising on Meat Demand. American Journal of Agricultural Economics, 77(4):969-979.

Che, H., Sudhir, K., and Seetharaman, P. B. (2007). Bounded Rationality in Pricing Under State-Dependent Demand: Do Firms Look Ahead, and if So, How Far? Journal of Marketing Research, 44(3):434-449.

Choi, S. C. and Coughlan, A. T. (2006). Private label positioning: Quality versus feature differentiation from the national brand. Journal of Retailing, 82(2):79-93.

Dubé, J.-P. and Manchanda, P. (2005). Differences in Dynamic Brand Competition Across Markets: An Empirical Analysis. Marketing Science, 24(1):81-95.

Eastlack, J. O. and Rao, A. G. (1986). Modeling Response to Advertising and Pricing Changes for "V-8" Cocktail Vegetable Juice. Marketing Science, 5(3):245-259. 
Erdem, T. and Chang, S. R. (2012). A cross-category and cross-country analysis of umbrella branding for national and store brands. Journal of the Academy of Marketing Science, 40(1):86-101.

Horowitz, I. (2000). An option-pricing look at the introduction of private labels. Journal of the Operational Research Society, 51(2):221-230.

Huang, Y. and Huddleston, P. (2009). Retailer premium own-brands: creating customer loyalty through own-brand products advantage. International Journal of Retail 83 Distribution Management, 37(11):975-992.

Karp, H. (2012). Store Brands Step Up Their Game, and Prices.

Karray, S. and Martín-Herrán, G. (2008). Investigating the Relationship Between Advertising and Pricing in a Channel with Private Label Offering: A Theoretic Model. Review of Marketing Science, 6(1).

Karray, S. and Martín-Herrán, G. (2009). A dynamic model for advertising and pricing competition between national and store brands. European Journal of Operational Research, 193(2):451-467.

Karray, S. and Zaccour, G. (2005). A Differential Game of Advertising for National and Store Brands. In Haurie, A. and Zaccour, G., editors, Dynamic Games: Theory and Applications, pages 213-229. Springer US.

Karray, S. and Zaccour, G. (2006). Could co-op advertising be a manufacturer's counterstrategy to store brands? Journal of Business Research, 59(9):1008-1015.

Kumar, N. and Steenkamp, J.-b. E. M. (2007). Private Label Strategy: How to Meet the Store Brand Challenge. Harvard Business Review Press, 1 edition.

Levy, S. and Gendel-Guterman, H. (2012). Does advertising matter to store brand purchase intention? A conceptual framework. Journal of Product \& Brand Management, 21(2):8997.

McDonald, S. and Wren, C. (2012). Informative Brand Advertising and Pricing Strategies in Internet Markets with Heterogeneous Consumer Search. International Journal of the Economics of Business, 19(1):103-117.

Mills, D. E. (1999). Private labels and manufacturer counterstrategies. European Review of Agricultural Economics, 26(2):125-145. 
Narasimhan, C. and Wilcox, R. T. (1998). Private Labels and the Channel Relationship: A Cross Category Analysis. The Journal of Business, 71(4):573-600.

Patti, C. H. and Fisk, R. P. (1982). National advertising, brands, and channel control: An historical perspective with contemporary options. Journal of the Academy of Marketing Science, 10(1):90-108.

Raju, J. S., Sethuraman, R., and Dhar, S. K. (1995). The Introduction and Performance of Store Brands. Management Science, 41(6):957-978.

Raju, J. S., Srinivasan, V., and Lal, R. (1990). The Effects of Brand Loyalty on Competitive Price Promotional Strategies. Management Science, 36(3):276-304.

Roberts, M. J. and Samuelson, L. (1988). An Empirical Analysis of Dynamic, Nonprice Competition in an Oligopolistic Industry. The RAND Journal of Economics, 19(2).

Sayman, S., Hoch, S. J., and Raju, J. S. (2002). Positioning of Store Brands. Marketing Science, 21(4):378-397.

Sethuraman, R. (2009). Assessing the External Validity of Analytical Results from National Brand and Store Brand Competition Models. Marketing Science, 28(4):759-781.

Sudhir, K. (2001). Structural Analysis of Manufacturer Pricing in the Presence of a Strategic Retailer. Marketing Science, 20(3):244-264.

Vilcassim, N. J., Kadiyali, V., and Chintagunta, P. K. (1999). Investigating Dynamic Multifirm Market Interactions in Price and Advertising. Management Science, 45(4):499-518.

Webster, F. E. (2000). Understanding the Relationships among Brands, Consumers, and Resellers. Journal of the Academy of Marketing Science, 28(1):17-23.

\section{A List of Mathematical Symbols and Notation}

SB: Store Brand

NB: National Brand

$p_{S}$ : the retail price of the SB good

$p_{N}$ : the retail price of the NB good 
$A_{S}$ : the market size of the $\mathrm{SB}$, also referred to as the advertising effort exerted by the SB $A_{N}$ : the market size of the $\mathrm{NB}$, also referred to as the advertising effort exerted by the NB $\underline{A_{N}}:$ the base market size for the NB good

$\beta$ : the fraction of the NB sales that are retained by the $\mathrm{SB}$, retailer, we assume $\beta \in(0,1)$

$\alpha_{S}$ : the advertising spillover from the NB to the SB, we assume $\alpha_{S} \in(0,1)$

$\alpha_{N}$ : the advertising spillover from the SB to the NB, we assume $\alpha_{N} \in(-1,1) ; \alpha_{N}<0$ models a competitive advertising environment and $\alpha_{N}>0$ models a complementary advertising environment

$a_{S}:$ the negative effect of $p_{S}$ on demand for the SB good

$a_{N}$ : the negative effect of $p_{N}$ on demand for the NB good

$b_{S}$ : the positive effect of $p_{N}$ on demand for the SB good

$b_{N}$ : the positive effect of $p_{S}$ on demand for the NB good

$c_{S}:$ the cost of advertising effort exerted by the SB

$c_{N}$ : the cost of advertising effort exerted by the NB

$\pi_{S}\left(A_{S}, p_{S} \mid A_{N}, p_{N}\right):$ the profit function of the SB

$\pi_{N}\left(A_{N}, p_{N}\right):$ the profit function of the NB

\section{B Mathematical Appendix}

In this appendix we provide the details of the results presented in Section 3.

\section{B.1 Advertising Only Case}

$$
\begin{aligned}
\pi_{S}\left(A_{S} \mid A_{N}\right)= & p_{S}\left(A_{S}+\alpha_{S}\left(A_{N}+\underline{A_{N}}\right)-a_{S} p_{S}+b_{S} p_{N}\right) \\
& +\beta p_{N}\left(A_{N}+\underline{A_{N}}+\alpha_{N} A_{S}-a_{N} p_{N}+b_{N} p_{S}\right) \\
& -c_{S}\left[\left(A_{S}+\alpha_{S}\left(A_{N}+\underline{A_{N}}\right)\right)^{2}-\left(\alpha_{S}\left(A_{N}+\underline{A_{N}}\right)\right)^{2}\right] .
\end{aligned}
$$


Its FOC leads to the profit maximizer $A_{S}^{*}$ :

$$
\begin{aligned}
\frac{d \pi_{S}\left(A_{S} \mid A_{N}\right)}{d A_{S}} & =p_{S}+\beta \alpha_{N} p_{N}-2 c_{S}\left(A_{S}+\alpha_{S}\left(A_{N}+\underline{A_{N}}\right)\right)=0 \\
\Longrightarrow A_{S}^{*} & =\frac{p_{S}+\beta \alpha_{N} p_{N}}{2 c_{S}}-\alpha_{S}\left(A_{N}+\underline{A_{N}}\right)
\end{aligned}
$$

In (17) $A_{S}^{*}$ is an interior point solution, including non-negativity constraint on $A_{S}$ we have:

$$
A_{S}^{*}\left(A_{N}\right)= \begin{cases}\frac{p_{S}+\beta \alpha_{N} p_{N}}{2 c_{S}}-\alpha_{S}\left(A_{N}+\underline{A_{N}}\right) & \text { if } \frac{p_{S}+\beta \alpha_{N} p_{N}}{2 \alpha_{S} c_{S}} \geq A_{N}+\underline{A_{N}} \\ 0 & \text { otherwise. }\end{cases}
$$

Note that we know the interior point is a maximum as $\pi_{S}\left(A_{S} \mid A_{N}\right)$ is concave, $\frac{d^{2} \pi_{S}\left(A_{S} \mid A_{N}\right)}{d A_{S}^{2}}=$ $-2 c_{S} \leq 0$.

Using (18), we may rewrite $\pi_{N}\left(A_{N}\right)$ as:

$$
\begin{gathered}
\pi_{N}\left(A_{N}\right)=(1-\beta) p_{N}\left(A_{N}+\underline{A_{N}}+\alpha_{N}\left(\frac{p_{S}+\beta \alpha_{N} p_{N}}{2 \alpha_{S}}-\alpha_{S}\left(A_{N}+\underline{A_{N}}\right)\right)-a_{N} p_{N}+b_{N} p_{S}\right) \\
-c_{N}\left[\left(A_{N}+\underline{A_{N}}\right)^{2}-{\underline{A_{N}}}^{2}\right] .
\end{gathered}
$$

We now find the FOC for $\pi_{N}\left(A_{N}\right)$ :

$$
\begin{aligned}
& \frac{d \pi_{N}\left(A_{N}\right)}{d A_{N}}(1-\beta) p_{N}\left(1-\alpha_{N} \alpha_{S}\right)-2 c_{N}\left(A_{N}+\underline{A_{N}}\right)=0 \\
& \Longrightarrow A_{N}^{*}=\frac{(1-\beta) p_{N}\left(1-\alpha_{N} \alpha_{S}\right)}{2 c_{N}}-\underline{A_{N}} .
\end{aligned}
$$

First note that $\pi_{N}\left(A_{N}\right)$ is concave, $\frac{d^{2} \pi_{N}\left(A_{N}\right)}{d A_{N}^{2}}=-2 c_{N} \leq 0$. For $A_{N}^{*}$ to be non-negative, it

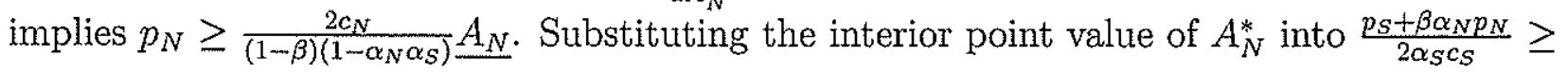
$A_{N}+\underline{A_{N}}$, the condition of interior $A_{S}^{*}$ in (18), we have:

$$
\begin{aligned}
\frac{p_{S}+\beta \alpha_{N} p_{N}}{2 \alpha_{S} c_{S}} & \geq \frac{(1-\beta) p_{N}\left(1-\alpha_{N} \alpha_{S}\right)}{2 c_{N}} \\
\Longrightarrow \frac{p_{S}}{p_{N}} & \geq(1-\beta) \alpha_{S}\left(1-\alpha_{N} \alpha_{S}\right) \frac{c_{S}}{c_{N}}-\beta \alpha_{N}
\end{aligned}
$$

Using the notation defined in Section 3.2, we let $\bar{p}_{N}=\frac{2 c_{N}}{(1-\beta)\left(1-\alpha_{N} \alpha_{S}\right)} A_{N}$ and $\phi=(1-\beta) \alpha_{S}(1-$ $\left.\alpha_{N} \alpha_{S}\right) \frac{c_{S}}{c_{N}}-\beta \alpha_{N}$. For each advertising decision, $A_{S}^{*}$ and $A_{N}^{*}$, we have four possible scenarios: both solutions are interior, only one of them is interior, or none of them are interior. They are defined with respect to the relationship between $p_{N}$ and $\bar{p}_{N}$, and independently between 
$\frac{p_{S}}{p_{N}}$ and $\phi$

$$
A_{N}^{*}= \begin{cases}\frac{(1-\beta)\left(1-\alpha_{N} \alpha_{S}\right)}{2} \cdot \frac{p_{N}}{c_{N}}-\underline{A_{N}} & \text { if } p_{N} \geq \bar{p}_{N} \text { and } \frac{p_{S}}{p_{N}} \geq \phi \\ \frac{1-\beta}{2} \cdot \frac{p_{N}}{c_{N}}-\underline{A_{N}} & \text { if } p_{N} \geq \bar{p}_{N} \text { and } \frac{p_{S}}{p_{N}}<\phi \\ 0 & \text { if } p_{N}<\bar{p}_{N} \text { and } \frac{p_{S}}{p_{N}} \geq \phi \\ 0 & \text { otherwise }\end{cases}
$$

Note that the second case of (28) follows from $A_{S}=0$ meaning the profit of the NB is $\pi_{N}\left(A_{N}\right)=(1-\beta) p_{N}\left(A_{N}+\underline{A_{N}}+a_{N} p_{N}+b_{N} p_{S}\right)-c_{N}\left[\left(A_{N}+\underline{A_{N}}\right)^{2}-{\underline{A_{N}}}^{2}\right]$. Using FOC we have:

$$
\begin{aligned}
\frac{d \pi_{N}\left(A_{N}\right)}{d A_{N}} & =(1-\beta) p_{N}-2 c_{N}\left(A_{N}+\underline{A_{N}}\right)=0 \\
\Longrightarrow A_{N}^{*} & =\frac{(1-\beta) p_{N}}{2 c_{N}}-\underline{A_{N}} .
\end{aligned}
$$

Note that in this case $\frac{d^{2} \pi_{N}\left(A_{N}\right)}{d A_{N}^{2}}=-1<0$ meaning that $\pi_{N}\left(A_{N}\right)$ is concave.

Similarly for $A_{S}^{*}$ we have:

$$
A_{S}^{*}= \begin{cases}\frac{1}{2} \frac{p_{S}}{c_{S}}+\frac{\beta \alpha_{N}}{2} \frac{p_{N}}{c_{S}}-\alpha_{S} \frac{(1-\beta)\left(1-\alpha_{N} \alpha_{S}\right)}{2} \frac{p_{N}}{c_{N}} & \text { if } p_{N} \geq \bar{p}_{N} \text { and } \frac{p_{S}}{p_{N}} \geq \phi \\ 0 & \text { if } p_{N} \geq \bar{p}_{N} \text { and } \frac{p_{S}}{p_{N}}<\phi \\ \frac{1}{2} \frac{p_{S}}{c_{S}}+\frac{\beta \alpha_{N}}{2} \frac{p_{N}}{c_{S}}-\alpha_{S} \underline{A_{N}} & \text { if } p_{N}<\bar{p}_{N} \text { and } \frac{p_{S}}{p_{N}} \geq \phi \\ 0 & \text { otherwise }\end{cases}
$$

Note that the third case of (29) follows from $A_{N}=0$ meaning the profit of the SB is $\pi_{S}\left(A_{S}\right)=$ $p_{S}\left(A_{S}+\alpha_{S} \underline{A_{N}}-a_{S} p_{S}+b_{S} p_{N}\right)+\beta p_{N}\left(\underline{A_{N}}+\alpha_{N} A_{S}-a_{N} p_{N}+b_{N} p_{S}\right)-c_{S}\left[\left(A_{S}+\alpha_{S} \underline{A_{N}}\right)^{2}-\left(\alpha_{S} \underline{A_{N}}\right)^{2}\right]$ Using FOC we have:

$$
\begin{aligned}
& \frac{d \pi_{S}\left(A_{S}\right)}{d A_{S}}=p_{S}+\beta \alpha_{N} p_{N}-2 c_{S}\left(A_{S}+\alpha_{S} \underline{A_{N}}\right)=0 \\
& \Longrightarrow A_{S}^{*}=\frac{p_{S}+\beta \alpha_{N} p_{N}}{2 c_{S}}-\alpha_{S} \underline{A_{N}} .
\end{aligned}
$$

Just as with the other cases, $\frac{d^{2} \pi_{S}\left(A_{S}\right)}{d A_{S}^{2}}=-2 c_{S} \leq 0$ meaning that $\pi_{S}\left(A_{S}\right)$ is concave.

\section{B.2 Pricing Only Case}

$$
\begin{aligned}
\pi_{S}\left(p_{S} \mid p_{N}\right)= & p_{S}\left(A_{S}+\alpha_{S}\left(A_{N}+\underline{A_{N}}\right)-a_{S} p_{S}+b_{S} p_{N}\right) \\
& +\beta p_{N}\left(A_{N}+\underline{A_{N}}+\alpha_{N} A_{S}-a_{N} p_{N}+b_{N} p_{S}\right) \\
& -c_{S}\left[\left(A_{S}+\alpha_{S}\left(A_{N}+\underline{A_{N}}\right)\right)^{2}-\left(\alpha_{S}\left(A_{N}+\underline{A_{N}}\right)\right)^{2}\right] .
\end{aligned}
$$


We now explore the FOC of the SB profit function assuming advertising decisions are fixed.

$$
\begin{aligned}
\frac{d \pi_{S}\left(p_{S} \mid p_{N}\right)}{d p_{S}} & =A_{S}+\alpha_{S}\left(A_{N}+\underline{A_{N}}\right)-a_{S} p_{S}+b_{S} p_{N}-a_{S} p_{S}+\beta p_{N} b_{N}=0 \\
\Longrightarrow p_{S}^{*} & =\frac{A_{S}+\alpha_{S}\left(A_{N}+\underline{A_{N}}\right)+b_{S} p_{N}+\beta p_{N} b_{N}}{2 a_{S}}
\end{aligned}
$$

First note that $\frac{d^{2} \pi_{S}\left(p_{S} \mid p_{N}\right)}{d p_{S}^{2}}=-2 a_{S}$ which means $\pi_{S}\left(p_{S} \mid p_{N}\right)$ is concave in $p_{S}$. Note that all of the terms in the right hand side of $(22)$ are positive, so the interior solution $p_{S}^{*}\left(p_{N}\right)$ is always feasible.

We now write $\pi_{N}\left(p_{N}\right)$, given $p_{S}^{*}\left(p_{N}\right)$ :

$$
\begin{aligned}
\pi_{N}\left(p_{N}\right)= & (1-\beta) p_{N}\left(A_{N}+\underline{A_{N}}+\alpha_{N} A_{S}-a_{N} p_{N}+b_{N}\left(\frac{A_{S}+\alpha_{S}\left(A_{N}+\underline{A_{N}}\right)+b_{S} p_{N}+\beta p_{N} b_{N}}{2 a_{S}}\right)\right) \\
& -c_{N}\left[\left(A_{N}+\underline{A_{N}}\right)^{2}-\underline{A_{N}}{ }^{2}\right] . \\
\frac{d \pi_{N}\left(p_{N}\right)}{d p_{N}}= & (1-\beta)\left(A_{N}+\underline{A_{N}}+\alpha_{N} A_{S}-a_{N} p_{N}+\frac{b_{N}}{2 a_{S}}\left(A_{S}+\alpha_{S}\left(A_{N}+\underline{A_{N}}\right)+b_{S} p_{N}+\beta p_{N} b_{N}\right)\right) \\
& +(1-\beta) p_{N}\left(-a_{N}+\frac{b_{N}}{2 a_{S}}\left(b_{S}+\beta b_{N}\right)\right)=0 \\
\Longrightarrow p_{N}^{*}= & \frac{A_{N}+\underline{A_{N}}+\alpha_{N} A_{S}+\frac{b_{N}}{2 a_{S}}\left(A_{S}+\alpha_{S}\left(A_{N}+\underline{A_{N}}\right)\right)}{2\left[a_{N}-\frac{b_{N}}{2 a_{S}}\left(b_{S}+\beta b_{N}\right)\right]}
\end{aligned}
$$

Note that $\frac{d^{2} \pi_{N}\left(p_{N}\right)}{d p_{N}^{2}}=2(1-\beta)\left(\frac{b_{N}}{2 a_{S}}\left(b_{S}+\beta b_{N}\right)-a_{N}\right)$, in order to ensure concavity we must have $a_{N} \geq \frac{b_{N}}{2 a_{S}}\left(b_{S}+\beta b_{N}\right)$. The numerator of $p_{N}^{*}$ is always positive, only the denominator may be negative in the case $a_{N}<\frac{b_{N}}{2 a_{S}}\left(b_{S}+\beta b_{N}\right)$, in order to ensure $p_{N}^{*}$ is non-negative we have add the condition that $a_{N}>\frac{b_{N}}{2 a_{S}}\left(b_{S}+\beta b_{N}\right)$ and to avoid division by zero, which is stronger than the concavity condition. As discussed in Section 3.3, we do not consider the case of $p_{N}^{*} \leq 0$ even when $\alpha_{N}$ is negative, as this would imply a SB may advertise a NB out of existence.

\section{B.3 General Case}

$$
\begin{aligned}
\pi_{S}\left(A_{S}, p_{S} \mid A_{N}, p_{N}\right)= & p_{S}\left(A_{S}+\alpha_{S}\left(A_{N}+\underline{A_{N}}\right)-a_{S} p_{S}+b_{S} p_{N}\right) \\
& +\beta p_{N}\left(A_{N}+\underline{A_{N}}+\alpha_{N} A_{S}-a_{N} p_{N}+b_{N} p_{S}\right) \\
& -c_{S}\left[\left(A_{S}+\alpha_{S}\left(A_{N}+\underline{A_{N}}\right)\right)^{2}-\left(\alpha_{S}\left(A_{N}+\underline{A_{N}}\right)\right)^{2}\right] .
\end{aligned}
$$




$$
\begin{gathered}
\nabla \pi_{S}\left(A_{S}, p_{S} \mid A_{N}, p_{N}\right)=\left[\begin{array}{c}
\frac{\partial \pi_{S}\left(A_{S}, p_{S} \mid A_{N}, p_{N}\right)}{\partial A_{S}} \\
\frac{\partial \pi_{S}\left(A_{S}, p_{S} \mid A_{N}, p_{N}\right)}{\partial p_{S}}
\end{array}\right] \\
=\left[\begin{array}{c}
p_{S}+\beta p_{N} \alpha_{N}-2 c_{S}\left(A_{S}+\alpha_{S}\left(A_{N}+\underline{A_{N}}\right)\right) \\
\left(A_{S}+\alpha_{S}\left(A_{N}+\underline{A_{N}}\right)-2 a_{S} p_{S}+b_{S} p_{N}\right)+\beta p_{N} b_{N}
\end{array}\right] \\
\nabla^{2} \pi_{S}\left(A_{S}, p_{S} \mid A_{N}, p_{N}\right)=\left[\begin{array}{cc}
\frac{\partial^{2} \pi_{S}\left(A_{S}, p_{S} \mid A_{N}, p_{N}\right)}{\partial A_{S}^{2}} & \frac{\partial^{2} \pi_{S}\left(A_{S}, p_{S} \mid A_{N}, p_{N}\right)}{\partial A_{S} \partial p_{S}} \\
\frac{\partial^{2} \pi_{S}\left(A_{S}, p_{S} \mid A_{N}, p_{N}\right)}{\partial p_{S} \partial A_{S}} & \frac{\partial^{2} \pi_{S}\left(A_{S}, p_{S} \mid A_{N}, p_{N}\right)}{\partial p_{S}^{2}}
\end{array}\right] \\
=\left[\begin{array}{cc}
-2 c_{S} & 1 \\
1 & -2 a_{S}
\end{array}\right]
\end{gathered}
$$

For the SB's best response to the NB's actions $A_{N}$ and $p_{N}$, we solve for FOCs, $\nabla \pi_{S}\left(A_{S}, p_{S} \mid A_{N}, p_{N}\right)=$ $\left[\begin{array}{l}0 \\ 0\end{array}\right]$, to find $A_{S}^{*}\left(A_{N}, p_{N}\right)$ and $p_{S}^{*}\left(A_{N}, p_{N}\right)$ :

$$
\begin{aligned}
p_{S}+\beta p_{N} \alpha_{N}-2 c_{S}\left(A_{S}+\alpha_{S}\left(A_{N}+\underline{A_{N}}\right)\right) & =0 \\
\left(A_{S}+\alpha_{S}\left(A_{N}+\underline{A_{N}}\right)-2 a_{S} p_{S}+b_{S} p_{N}\right)+\beta p_{N} b_{N} & =0
\end{aligned}
$$

$$
\begin{aligned}
& \Longrightarrow \\
& p_{S}=2 c_{S}\left(A_{S}+\alpha_{S}\left(A_{N}+\underline{A_{N}}\right)\right)-\beta p_{N} \alpha_{N} \\
& A_{S}=-\beta p_{N} b_{N}-\alpha_{S}\left(A_{N}+\underline{A_{N}}\right)+2 a_{S} \cdot\left(2 c_{S}\left(A_{S}+\alpha_{S}\left(A_{N}+\underline{A_{N}}\right)\right)-\beta p_{N} \alpha_{N}\right)-\beta p_{N} \alpha_{N}-b_{S} p_{N} \\
& A_{S}\left(1-4 a_{S} c_{S}\right)=-\beta p_{N} b_{N}-\alpha_{S}\left(A_{N}+\underline{A_{N}}\right)+4 a_{S} c_{S}\left(\alpha_{S}\left(A_{N}+\underline{A_{N}}\right)\right)-2 a_{S} \beta p_{N} \alpha_{N}-\beta p_{N} \alpha_{N}-b_{S} p_{N} \\
& =\left(-b_{S}-\beta b_{N}-2 \beta a_{S} \alpha_{N}\right) p_{N}+\alpha_{S}\left(4 a_{S} c_{S}-1\right)\left(A_{N}+\underline{A_{N}}\right) \\
& A_{S}^{*}\left(A_{N}, p_{N}\right)= \begin{cases}\frac{b_{S}+\beta b_{N}+2 \beta a_{S} \alpha_{N}}{4 a_{S} c_{S}-1} p_{N}-\alpha_{S}\left(A_{N}+\underline{A_{N}}\right) & \text { if } \frac{b_{S}+\beta b_{N}+2 \beta a_{S} \alpha_{N}}{\alpha_{S}\left(4 a_{S} c_{S}-1\right)} p_{N}>A_{N}+\underline{A_{N}} \\
0 & \text { otherwise. }\end{cases}
\end{aligned}
$$




$$
\begin{aligned}
p_{S} & =2 c_{S}\left(\frac{b_{S}+\beta b_{N}+2 \beta a_{S} \alpha_{N}}{4 a_{S} c_{S}-1} p_{N}-\alpha_{S}\left(A_{N}+\underline{A_{N}}\right)+\alpha_{S}\left(A_{N}+\underline{A_{N}}\right)\right)-\beta p_{N} \alpha_{N} \\
& =\left(\frac{2 c_{S} b_{S}+2 \beta c_{S} b_{N}+4 \beta c_{S} a_{S} \alpha_{N}}{4 a_{S} c_{S}-1}-\beta \alpha_{N}\right) p_{N} \\
& =\frac{2 c_{S} b_{S}+2 \beta c_{S} b_{N}+4 \beta c_{S} a_{S} \alpha_{N}-4 a_{S} c_{S} \beta \alpha_{N}+\beta \alpha_{N}}{4 a_{S} c_{S}-1} p_{N} \\
p_{S}^{*}\left(A_{N}, p_{N}\right) & = \begin{cases}\frac{\beta \alpha_{N}+2 b_{S} c_{S}+2 \beta b_{N} c_{S}}{4 a_{S} c_{S}-1} p_{N} & \text { if } \frac{b_{S}+\beta b_{N}+2 \beta a_{S} \alpha_{N}}{\alpha_{S}\left(4 a_{S} c_{S}-1\right)} p_{N}>A_{N}+\underline{A_{N}} \\
0 & \text { otherwise. }\end{cases}
\end{aligned}
$$

Given the optimal values of $p_{S}^{*}\left(A_{N}, p_{N}\right)$ and $A_{S}^{*}\left(A_{N}, p_{N}\right)$ we rewrite $\pi_{N}\left(A_{N}, p_{N}\right)$ as:

$$
\begin{aligned}
& \pi_{N}\left(A_{N}, p_{N}\right)=(1-\beta) p_{N}\left(A_{N}+\underline{A_{N}}+\alpha_{N}\left(\frac{b_{S}+\beta b_{N}+2 \beta a_{S} \alpha_{N}}{4 a_{S} c_{S}-1} p_{N}-\alpha_{S}\left(A_{N}+\underline{A_{N}}\right)\right)\right. \\
& \left.-a_{N} p_{N}+b_{N} p_{N} \frac{\beta \alpha_{N}+2 b_{S} c_{S}+2 \beta b_{N} c_{S}}{4 a_{S} c_{S}-1}\right)-c_{N}\left[\left(A_{N}+\underline{A_{N}}\right)^{2}-\underline{A_{N}}{ }^{2}\right] \text {. } \\
& \nabla \pi_{N}\left(A_{N}, p_{N}\right)=\left[\begin{array}{c}
\frac{\partial \pi_{N}\left(A_{N}, p_{N}\right)}{\partial A_{N}} \\
\frac{\partial \pi_{N}\left(A_{N}, p_{N}\right)}{\partial p_{N}}
\end{array}\right] \\
& =\left[\begin{array}{c}
(1-\beta) p_{N}\left(1-\alpha_{N} \alpha_{S}\right)-2 c_{N} \cdot\left(A_{N}+\underline{A_{N}}\right) \\
(1-\beta)\left(A_{N}+\underline{A_{N}}+\alpha_{N}\left(\frac{b_{S}+\beta b_{N}+2 \beta a_{S} \alpha_{N}}{4 a_{S} c_{S}-1} p_{N}-\alpha_{S}\left(A_{N}+\underline{A_{N}}\right)\right.\right. \\
\left.-a_{N} p_{N}+b_{N} p_{N} \frac{\beta \alpha_{N}+2 b_{S} c_{S}+2 \beta b_{N} c_{S}}{4 a_{S} c_{S}-1}\right) \\
+(1-\beta) p_{N}\left(\alpha_{N} \frac{b+\beta b_{N}+2 \beta a_{S} \alpha_{N}}{4 a_{S} c_{S}-1}-a_{N}+b_{N} \frac{\beta \alpha_{N}+2 b_{S} c_{S}+2 \beta b_{N} c_{S}}{4 a_{S} c_{S}-1}\right)
\end{array}\right] \\
& \begin{aligned}
\nabla^{2} \pi_{N}\left(A_{N}, p_{N}\right) & =\left[\begin{array}{ll}
\frac{\partial^{2} \pi_{N}\left(A_{N}, p_{N}\right)}{\partial A_{N}^{2}} & \frac{\partial^{2} \pi_{N}\left(A_{N}, p_{N}\right)}{\partial A_{N} \partial p_{N}} \\
\frac{\partial^{2} \pi_{N}\left(A_{N}, p_{N}\right)}{\partial p_{N} \partial A_{N}} & \frac{\partial^{2} \pi_{N}\left(A_{N}, p_{N}\right)}{\partial p_{N}^{2}}
\end{array}\right] \\
& =\left[\begin{array}{cc}
-2 c_{N} & (1-\beta)\left(1-\alpha_{N} \alpha_{S}\right) \\
(1-\beta)\left(1-\alpha_{N} \alpha_{S}\right) & 2(1-\beta)\left(\alpha_{N} \frac{b_{S}+\beta b_{N}+2 \beta a_{S} \alpha_{N}}{4 a_{S} c_{S}-1}-a_{N}+b_{N} \frac{\beta \alpha_{N}+2 b_{S} c_{S}+2 \beta b_{N} c_{S}}{4 a_{S} c_{S}-1}\right)
\end{array}\right] \\
& =\left[\begin{array}{cc}
-2 c_{N} \\
\left(1-\alpha_{N} \alpha_{S}\right)(1-\beta) \\
\left(1-\alpha_{N} \alpha_{S}\right)(1-\beta) & -2(1-\beta)\left(a_{N}-\frac{\alpha_{N}\left(b_{S}+b_{N} \beta+2 a_{S} \alpha_{N} \beta\right)+b_{N}\left(\alpha_{N} \beta+2 b_{S} c_{S}+2 b_{N} \beta c_{S}\right)}{4 a_{S} c_{S}-1}\right)
\end{array}\right]
\end{aligned}
\end{aligned}
$$

In order to ensure the FOCs, $\nabla \pi_{N}\left(A_{N}, p_{N}\right)=\left[\begin{array}{l}0 \\ 0\end{array}\right]$, minimize $\pi_{N}\left(A_{N}, p_{N}\right)$, we have 
to ensure $\nabla^{2} \pi_{N}\left(A_{N}, p_{N}\right)$ is negative semidefinite, i.e., $\left|\nabla^{2} \pi_{N}\left(A_{N}, p_{N}\right)\right| \leq 0$. Formally this means that:

$$
c_{N} \geq \frac{\left(1-\alpha_{N} \alpha_{S}\right)^{2}(1-\beta)}{4\left(a_{N}-\frac{\alpha_{N}\left(b_{S}+b_{N} \beta+2 a_{S} \alpha_{N} \beta\right)+b_{N}\left(\alpha_{N} \beta+2 b_{S} c_{S}+2 b_{N} \beta c_{S}\right)}{4 a_{S} c_{S}-1}\right)} .
$$

Given this condition, we consider $\nabla \pi_{N}\left(A_{N}, p_{N}\right)=\left[\begin{array}{l}0 \\ 0\end{array}\right]$ :

$$
\begin{aligned}
&(1-\beta) p_{N}\left(1-\alpha_{N} \alpha_{S}\right)-2 c_{N} \cdot\left(A_{N}+\underline{A_{N}}\right)=0 \\
&(1-\beta)\left(A_{N}+\underline{A_{N}+\alpha_{N}\left(\frac{b_{S}+\beta b_{N}+2 \beta a_{S} \alpha_{N}}{4 a_{S} c_{S}-1} p_{N}-\alpha_{S}\left(A_{N}+\underline{A_{N}}\right)\right)}\right. \\
&\left.-a_{N} p_{N}+b_{N} p_{N} \frac{\beta \alpha_{N}+2 b_{S} c_{S}+2 \beta b_{N} c_{S}}{4 a_{S} c_{S}-1}\right)=0 \\
&+(1-\beta) p_{N}\left(\alpha_{N} \frac{b_{S}+\beta b_{N}+2 \beta a_{S} \alpha_{N}}{4 a_{S} c_{S}-1}-a_{N}+b_{N} \frac{\beta \alpha_{N}+2 b_{S} c_{S}+2 \beta b_{N} c_{S}}{4 a_{S} c_{S}-1}\right)
\end{aligned}
$$

$$
\begin{aligned}
& \Longrightarrow \\
& p_{N}=\frac{2 c_{N}\left(A_{N}+\underline{A_{N}}\right)}{(1-\beta)\left(1-\alpha_{N} \alpha_{S}\right)}>0 \text { for } \beta, \alpha_{S} \in(0,1) \text { and } \alpha_{N} \in(-1,1) \\
& 0=\left(A_{N}+\underline{A_{N}}\right)(1-\beta)\left(1-\alpha_{N} \alpha_{S}\right) \\
& +2 p_{N}(1-\beta)\left(\alpha_{N} \frac{b_{S}+\beta b_{N}+2 \beta a_{S} \alpha_{N}}{4 a_{S} c_{S}-1}-a_{N}+b_{N} \frac{\beta \alpha_{N}+2 b_{S} c_{S}+2 \beta b_{N} c_{S}}{4 a_{S} c_{S}-1}\right) \\
& 0=\left(A_{N}+\underline{A_{N}}\right)(1-\beta)\left(1-\alpha_{N} \alpha_{S}\right) \\
& +\frac{4 c_{N}\left(A_{N}+\underline{A_{N}}\right)}{(1-\beta)\left(1-\alpha_{N} \alpha_{S}\right)}(1-\beta)\left(\alpha_{N} \frac{b_{S}+\beta b_{N}+2 \beta a_{S} \alpha_{N}}{4 a_{S} c_{S}-1}-a_{N}+b_{N} \frac{\beta \alpha_{N}+2 b_{S} c_{S}+2 \beta b_{N} c_{S}}{4 a_{S} c_{S}-1}\right) \\
& 0=\left(A_{N}+\underline{A_{N}}\right)\left[(1-\beta)\left(1-\alpha_{N} \alpha_{S}\right)\right. \\
& +\frac{4 c_{N}}{(1-\beta)\left(1-\alpha_{N} \alpha_{S}\right)}(1-\beta)\left(\alpha_{N} \frac{b_{S}+\beta b_{N}+2 \beta a_{S} \alpha_{N}}{4 a_{S} c_{S}-1}-a_{N}+b_{N} \frac{\beta \alpha_{N}+2 b_{S} c_{S}+2 \beta b_{N} c_{S}}{4 a_{S} c_{S}-1}\right) \\
& 0=\left(A_{N}+\underline{A_{N}}\right) \Delta
\end{aligned}
$$

where $\Delta=(1-\beta)\left(1-\alpha_{N} \alpha_{S}\right)+4 c_{N} \frac{2 a_{S} \beta \alpha_{N}^{2}+2 \beta \alpha_{N} b_{N}+b_{S} \alpha_{N}+2 \beta c_{S} b_{N}^{2}+2 b_{S} c_{S} b_{N}-a_{N}\left(4 a_{S} c_{S}-1\right)}{\left(4 a_{S} c_{S}-1\right)\left(1-\alpha_{N} \alpha_{S}\right)}$. Note that in an interior solution, $A_{N}=-A_{N}$, however as $\underline{A_{N}} \geq 0$ and $A_{N} \geq 0$ an interior solution is not feasible and only a corner solution exists. A corner solution is the only option for $A_{N} \in[0, \infty)$. As an interior stationary point exists and by construction $\pi_{N}\left(A_{N}, p_{N}\right)$ is concave, we know that $\pi_{N}\left(A_{N}, p_{N}\right)$ is increasing for all values of $A_{N}<-A_{N}$ and decreasing for all values $A_{N} \geq-A_{N}$, and thus $A_{N}^{*}=0, p_{N}^{*}=\frac{2 c_{N} A_{N}}{(1-\beta)\left(1-\alpha_{N} \alpha_{S}\right)}$ is the feasible point that maximizes $\pi_{N}\left(A_{N}, p_{N} \mid A_{S}^{*}, p_{S}^{*}\right)$. 


\section{Sensitivity Analysis on $\beta$}

The optimal decisions, pricing and advertising, determined in Section 3 may depend on the wholesale price that we model using an exogenously set parameter $\beta$. In this section we examine how these decisions change with $\beta$.

From Section 3.2 we know that when $p_{S}$ and $p_{N}$ are fixed, the optimal advertising decisions are:

$$
A_{N}^{*}= \begin{cases}\frac{(1-\beta)\left(1-\alpha_{N} \alpha_{S}\right)}{2} \cdot \frac{p_{N}}{c_{N}}-\underline{A_{N}} & \text { if } p_{N}>\bar{p}_{N} \text { and } \frac{p_{S}}{p_{N}}>\phi \\ \frac{1-\beta}{2} \cdot \frac{p_{N}}{c_{N}}-\underline{A_{N}} & \text { if } p_{N}>\bar{p}_{N} \text { and } \frac{p_{S}}{p_{N}} \leq \phi \\ 0 & \text { if } p_{N} \leq \bar{p}_{N} \text { and } \frac{p_{S}}{p_{N}}>\phi \\ 0 & \text { otherwise }\end{cases}
$$

and

$$
A_{S}^{*}=\left\{\begin{array}{ll}
\frac{1}{2} \frac{p_{S}}{c_{S}}+\frac{\beta \alpha_{N}}{2} \frac{p_{N}}{c_{S}}-\alpha_{S} \frac{(1-\beta)\left(1-\alpha_{N} \alpha_{S}\right)}{2} \frac{p_{N}}{c_{N}} & \text { if } p_{N}>\bar{p}_{N} \text { and } \frac{p_{S}}{p_{N}}>\phi \\
0 & \text { if } p_{N}>\bar{p}_{N} \text { and } \frac{p_{S}}{p_{N}} \leq \phi \\
\frac{1}{2} \frac{p S}{c_{S}}+\frac{\beta \alpha_{N}}{2} \frac{p_{N}}{c_{S}}-\alpha_{S} \underline{A_{N}} & \text { if } p_{N} \leq \bar{p}_{N} \text { and } \frac{p_{S}}{p_{N}}>\phi \\
0 & \text { otherwise }
\end{array} .\right.
$$

We take the derivative of each decision with respect to $\beta$ to determine how each will change with the wholesale price:

$$
\frac{\partial A_{N}^{*}}{\partial \beta}= \begin{cases}-\frac{\left(1-\alpha_{N} \alpha_{S}\right)}{2} \cdot \frac{p_{N}}{c_{N}} & \text { if } p_{N}>\bar{p}_{N} \text { and } \frac{p_{S}}{p_{N}}>\phi \\ -\frac{1}{2} \cdot \frac{p_{N}}{c_{N}} & \text { if } p_{N}>\bar{p}_{N} \text { and } \frac{p_{S}}{p_{N}} \leq \phi \\ 0 & \text { if } p_{N} \leq \bar{p}_{N} \text { and } \frac{p_{S}}{p_{N}}>\phi \\ 0 & \text { otherwise }\end{cases}
$$

and

$$
\frac{\partial A_{S}^{*}}{\partial \beta}=\left\{\begin{array}{lll}
\frac{\alpha_{N}}{2} \frac{p_{N}}{c_{S}}+\alpha_{S} \frac{\left(1-\alpha_{N} \alpha_{S}\right)}{2} \frac{p_{N}}{c_{N}} & \text { if } p_{N}>\bar{p}_{N} \text { and } \frac{p_{S}}{p_{N}}>\phi \\
0 & \text { if } p_{N}>\bar{p}_{N} \text { and } \frac{p_{S}}{p_{N}} \leq \phi \\
\frac{\alpha_{N}}{2} \frac{p_{N}}{c_{S}} & \text { if } p_{N} \leq \bar{p}_{N} \text { and } \frac{p_{S}}{p_{N}}>\phi \\
0 & \text { otherwise }
\end{array} .\right.
$$

From the equation (30) and (31) we note that the NB will decrease their advertising effort with $\beta$, i.e., lower wholesale price will lead to lower advertising effort. Conversely, as wholesale price decreases, $\beta$ increases, the SB will exert larger effort in the case advertising campaigns are complementary, $\alpha_{N}$ is positive. However, in the case advertising campaigns are competitive, the effect of $\beta$ on the SB advertising decision may depend on the relative magnitude between $\alpha_{N}$ and $\alpha_{S}$ among other parameters, i.e., whether the direct competing effect $\frac{\alpha_{N}}{2} \frac{p_{N}}{c_{S}}$ is stronger than indirect spillover effect $\alpha_{S} \frac{\left(1-\alpha_{N} \alpha_{S}\right)}{2} \frac{p_{N}}{c_{N}}$.

In the pricing only case we have: 


$$
p_{N}^{*}=\frac{A_{N}+\underline{A_{N}}+\alpha_{N} A_{S}+\frac{b_{N}}{2 a_{S}}\left[A_{S}+\alpha_{S}\left(A_{N}+\underline{A_{N}}\right)\right]}{2\left[a_{N}-\frac{b_{N}}{2 a_{S}}\left(b_{S}+\beta b_{N}\right)\right]}
$$

and

$$
p_{S}^{*}=\frac{A_{S}+\alpha_{S}\left(A_{N}+A_{N}\right)+b_{S} p_{N}^{*}+\beta p_{N}^{*} b_{N}}{2 a_{S}} .
$$

Looking at the partial derivatives we have:

$$
\frac{\partial p_{N}^{*}}{\partial \beta}=\frac{A_{N}+\underline{A_{N}}+\alpha_{N} A_{S}+\frac{b_{N}}{2 a_{S}}\left[A_{S}+\alpha_{S}\left(A_{N}+\underline{A_{N}}\right)\right]}{2\left[a_{N}-\frac{b_{N}}{2 a_{S}}\left(b_{S}+\beta b_{N}\right)\right]^{2}} \cdot \frac{b_{N}^{2}}{2 a_{s}}
$$

and

$$
\frac{\partial p_{S}^{*}}{\partial \beta}=\frac{b_{N}}{2 a_{S}}\left(p_{N}^{*}+\beta \frac{\text { partialp }}{\partial \beta}\right) .
$$

From equation (32) we note that $p_{N}$ is always increasing in $\beta$. The same relationship holds for $p_{S}$ as $p_{N}(\beta) \geq 0$ by condition (8), and thus $p_{S}$ is also increasing in $\beta$.

We finally consider the general case:

$$
\begin{aligned}
p_{N}^{*} & =\frac{2 c_{N} A_{N}}{(1-\beta)\left(1-\alpha_{N} \alpha_{S}\right)} \\
A_{N}^{*} & =0
\end{aligned}
$$

and

$$
\begin{aligned}
& A_{S}^{*}\left(A_{N}, p_{N}\right)= \begin{cases}\frac{b_{S}+\beta b_{N}+2 \beta a_{S} \alpha_{N}}{4 a_{S} c_{S}-1} p_{N}^{*}-\alpha_{S} \underline{A_{N}} & \text { if } \frac{b_{S}+\beta b_{N}+2 \beta a_{S} \alpha_{N}}{\alpha_{S}\left(4 a_{S} c_{S}-1\right)} p_{N}^{*}>\underline{A_{N}} \\
0 & \text { otherwise. }\end{cases} \\
& p_{S}^{*}\left(A_{N}, p_{N}\right)= \begin{cases}\frac{\beta \alpha_{N}+2 b_{S} c_{S}+2 \beta b_{N} c_{S}}{4 a_{S} c_{S}-1} p_{N}^{*} & \text { if } \frac{b_{S}+\beta b_{N}+2 \beta a_{S} \alpha_{N}}{\alpha_{S}\left(4 a_{S} c_{S}-1\right)} p_{N}^{*}>\underline{A_{N}} \\
0 & \text { otherwise. }\end{cases}
\end{aligned}
$$

Taking the partial derivatives of the equilibrium decisions with respect to $\beta$ we have:

$$
\frac{\partial p_{N}^{*}}{\partial \beta}=-\frac{2 c_{N} \underline{A}_{N}}{(1-\beta)^{2}\left(1-\alpha_{N} \alpha_{S}\right)}
$$

and

$$
\begin{aligned}
& \frac{\partial A_{S}^{*}}{\partial \beta}= \begin{cases}\frac{b_{S}+\beta b_{N}+2 \beta a_{S} \alpha_{N}}{4 a_{S} c_{S}-1} \frac{\partial p_{N}^{*}}{\partial \beta}+\frac{b_{N}+2 a_{S} \alpha_{N}}{4 a_{S} c_{S}-1} p_{N}^{*} & \text { if } \frac{b_{S}+\beta b_{N}+2 \beta a_{S} \alpha_{N}}{\alpha_{S}\left(4 a_{S} c_{S}-1\right)} p_{N}^{*}>\underline{A_{N}} \\
0 & \text { otherwise. }\end{cases} \\
& \frac{\partial p_{S}^{*}}{\partial \beta}= \begin{cases}\frac{\beta \alpha_{N}+2 b_{S} c_{S}+2 \beta b_{N} c_{S}}{4 a_{S} c_{S}-1} \frac{\partial p_{N}^{*}}{\partial \beta}+\frac{\alpha_{N}+2 b_{N} c_{S}}{4 a_{S} c_{S}-1} p_{N}^{*} & \text { if } \frac{b S+\beta b_{N}+2 \beta a_{S} \alpha_{N}}{\alpha_{S}\left(4 a_{S} c_{S}-1\right)} p_{N}^{*}>\underline{A_{N}} \\
0 & \text { otherwise. }\end{cases}
\end{aligned}
$$


From (34) we note that $p_{N}$ is decreasing in $\beta$ for the general hand. Things are not so obvious for the SB, $A_{S}$ and $p_{S}$ may increase or decrease with $\beta$ depending on the relationships between the terms. If the first term of each of the partials is greater than the second then, the optimal decisions decrease with $\beta$, otherwise they both increase with $\beta$. 